\title{
Influencia de los métodos de lectura en los hábitos de trabajo intelectual de los escolares
}

Pedro Hernández* Juan E. Jiménez

Universidad de La Laguna

\section{INTRODUCCION}

Tradicionalmente, la investigación que se ha dedicado a comprobar las ventajas de unos métodos de enseñanza de la lectura sobre otros, ha tomado como criterios de evaluación, la exactitud, la velocidad y la comprensión lectora (Jiménez y Hernández, 1986). Sin embargo, son desconocidas las investigaciones que se han interesado por analizar el efecto que tienen los distintos métodos de lectura en los hábitos o estrategias intelectuales de los alumnos. ¿Los alumnos que aprenden a leer por un método fónico o silábico desarrollan iguales hábitos intelectivos que los que aprenden por un método global-natural?

Entre los distintos métodos existentes, el debate se fundamenta entre los que defienden una orientación basada en la decodificación del significante frente a los que prefieren trabajar sobre el significado (Williams, 1965; Bond y Dykstra, 1967; Chall, 1967; Gibson y Levin, 1975).

Para poder obtener claridad sobre esta cuestión, es necesario contemplar antes cuáles son los objetivos de la lectura.

Un planteamiento educativo óptimo de la enseñanza de la lectura debe conseguir que el niño no solamente domine el desciframiento de los signos (significante) o los contenidos que se transmite (significado), considerados ambos como aspectos reproductivos, sino también en ser capaz de juzgar lo que se lee, de elaborar nuevas informaciones y de saber transferir las aprendidas a otras situaciones distintas, consideradas como aspectos productivos.

Por consiguiente, cabe preguntarse: ¿los métodos de lectura que se emplean actualmente en la escuela, consiguen esos objetivos?

Lo que sí parece evidente es que los objetivos de tipo reproductivo se consiguen a través de los distintos métodos de lectura, tal y como queda reflejado en numerosos informes y revisiones en la literatura (Bond y Dykstra, 1967; Weintraub et al., 1971-75, 1982; Otto et al., 1971-1973; Pflaum et al., 1980).

* Dirección de los autores: Pedro Hernández, ICE de la Universidad de La Laguna. Juan E. Jiménez, Departamento de Psicología de la Educación. Universidad de La Laguna. Tenerife. 
Se plantea, entonces, la necesidad de estudiar cómo pueden estar influyendo esas formas intruccionales en el desarrollo de las capacidades intelectuales de tipo productivo. Por ejemplo, las que estimulan la elaboración personal de la información. En este sentido, la información que es presentada al sujeto, sirve de punto de partida para ser transformada, o para asociarla, de forma que se obtenga una nueva información (Hernández y García, 1983a).

Para ello, se desarrolló longitudinalmente durante dos cursos, correspondientes al ciclo inicial de la EGB ( 6 a 8 años) sobre una muestra de 565 escolares que seguían el proceso de enseñanza-aprendizaje de la lectura, según distintos métodos.

A la hora de evaluar las habilidades de trabajo intelectual durante la lectura, se ha tomado como marco de referencia, la revisión de Rosenshine (1980) para la selección de las mismas. Así, se tuvieron en cuenta habilidades como poner un título al texto leído, realizar una síntesis o resumen, extraer las ideas principales y responder a cuestiones específicas del texto.

\section{METODO}

\section{Sujetos}

La muestra estaba formada por 565 escolares, de ambos sexos, procedentes de 29 aulas, de colegios públicos y privados y pertenecientes a estratos sociales y zonas de residencia diferentes.

Estaban distribuidos en tres grupos, según la orientación instruccional del maestro en la enseñanza de la lectura. El grupo primero correspondía al método global-natural $(n=247)$, el grupo segundo que sigue la instrucción silábica $(n=164)$ y el grupo tercero, perteneciente al método fónico $(n=154)$. La clasificación de los métodos vino dada por el análisis factorial derivado del instrumento «Diagnóstico Instruccional» de la lectura (Jiménez y Hernández, 1985).

La distribución de los escolares según sexo, nivel socioeconómico, tipo de colegio y zona de residencia está expresada en la tabla I.

Tabla I

Distribución de la muestra según sexo, nivel socioeconómico, tipo de colegio y zona de residencia

\begin{tabular}{|l|r|r|r|c|c|c|c|c|c|c|}
\hline \multirow{2}{*}{ Grupo } & \multicolumn{2}{|c|}{ Sexo } & \multicolumn{3}{c|}{ Nivel socioeconómico } & \multicolumn{2}{c|}{ Colegio } & \multicolumn{2}{c|}{ Zona de residencia } \\
\cline { 2 - 11 } & V & M & \multicolumn{1}{c|}{ B } & M & A & Publico & Privado & Rural & Urb. Per. & Urb. Cen. \\
\hline Global-Natural & 130 & 117 & 198 & 24 & 25 & 187 & 60 & 131 & 86 & 30 \\
Silábico & 92 & 72 & 124 & 23 & 17 & 135 & 29 & 44 & 91 & 28 \\
Fónico & 75 & 80 & 96 & 35 & 23 & 113 & 42 & 63 & 50 & 42 \\
\hline
\end{tabular}

\section{Procedimiento.}

\section{a) Diseño de investigación y variables utilizadas}

Se planificó la investigación a través de un diseño factorial $3 \times 2 \times 2$. Se trata de un diseño de factores cruzados intergrupo, ya que todos los niños no pasan por las mismas condiciones.

Los niveles de la variable método de la enseñanza de lectura eran: 1) globalnatural; 2) silábico y 3) fónico. 
1) global-natural: destaca el uso de unidades lingüísticas con significado en la instrucción lectora, siendo la frase la unidad básica que lo caracteriza. Se trabaja la observación, localización y simbolización de la frase que es expresada por los niños en su contexto natural, para luego llegar al análisis de los elementos lingüísticos más simples (fonemas y sillabas) a través de sucesivas descomposiciones analíticas de dichas estructuras complejas.

2) silábico: se insiste, desde el principio, en la decodificación del significante, especialmente la introducción de significantes escritos (sílabas), así como a nivel oral para ser identificados y reproducidos.

3) fónico: se concede también especial importancia a la decodificación del significante, siendo el fonema la unidad lingüistica sobre la cual recae la instrucción. Se trabaja el análisis de los componentes fónicos constituyentes de las palabras, y se estimula tanto la búsqueda de estos componentes como su reproducción gráfica.

La variable tipo de texto escrito viene definida por la naturaleza de la estructura presentada en el texto, y es de dos tipos:

1) narrativa: predomina el relato de una historia que incluye tres fases diferenciadas: estado de la situación inicial, nudo y desenlace.

2) descriptiva: la estructura del texto consiste en la exposición o explicación de un objeto o situación.

Se tuvo en cuenta la naturaleza de los textos, pues, en estas edades, resultan mucho más fáciles para los niños textos de tipo narrativo. En muchos países, la instrucción de la lectura es llevada a cabo mediante ese tipo de materiales (Kent, 1984). Sin embargo, a partir del tercer o cuarto curso, se empiezan a introducir con mayor frecuencia textos de tipo descriptivo (Alvermann y Boothby, 1982).

Por este motivo, en esta investigación, además de textos de tipo narrativo, también se introdujeron textos sencillos de naturaleza descriptiva, con el objeto de analizar mejor los efectos de transferencia de aprendizaje desde el material más familiar (narrativo) al menos familiar (descriptivo).

La variable tipo de presentación del texto, supone la manipulación del modo en que los niños han de contestar a los textos escritos. Esta variable presenta dos niveles:

1) texto presente: el niño lee primero el texto, y, a continuación, contesta a las preguntas pudiendo consultar el texto.

2) texto diferido: en este caso, el niño, una vez que ha finalizado la lectura del texto, contesta a las preguntas sin poder consultar el texto.

Las variables dependientes que se midieron estaban referidas a las habilidades que se evaluaban en cada una de las condiciones del estudio:

1) Dar un título al texto leído.

2) Capacidad de resumen: habilidad para expresar de forma breve las ideas más importantes o esenciales del texto leído.

3) Extracción de ideas principales: habilidad para discriminar y estructurar las ideas esenciales del texto leído.

4) Respuestas a cuestiones específicas.

\section{b) Instrumentos de evaluación}

Se construyeron cuatro textos, dos de carácter narrativo (TEXTN1 y TEXTN2) y dos de tipo descriptivo (TEXTD1 y TEXTD2). Cada texto contiene una hoja de respuestas adicional, donde vienen formuladas una serie de preguntas referidas al texto. En el Anexo I y II, se recoge el material empleado que incluye los textos utilizados y preguntas correspondientes a cada texto. 
Se emplearon los siguientes criterios de puntuación para cada una de las habilidades evaluadas:

1) poner un título: se asignaba sólo un punto si el título elegido respondía o tenía relación con el núcleo o argumento expuesto en el texto.

2) síntesis: en la valoración de la síntesis o resumen se asignaba puntuación solamente si el niño resumía la información contenida en el texto, sin llegar a reproducirla. Una vez cumplida esta condición, el criterio de corrección consistía en contabilizar las ideas principales y secundarias que destaca el sujeto en el trabajo de sintesis.

A la hora de determinar las diferencias entre idea principal y secundaria, los textos fueron asignados a una muestra de jueces. Se les indicó que señalasen qué partes del texto les resultaba las más importantes de destacar, y cuáles se subordinaban a éstas.

3) ideas principales: tomando como referencia lo hallado en la muestra de jueces, se asignaba un punto por cada idea principal descrita por el niño. En cada texto, se pedía al niño que escribiera los tres sucesos más importantes que dice el cuento.

4) respuestas específicas a las cuestiones formuladas en el texto: se asignaba un punto por cada pregunta que era contestada correctamente.

5) rendimiento global: se generó esta variable sumando las puntuaciones obtenidas en cada una de las habilidades lectoras comentadas hasta ahora.

\section{c) Descripción de los pasos realizados en la aplicación de las pruebas}

Cada una de las aulas que comprende la muestra $(n=29)$ fue dividida en cuatro grupos. La distribución de los textos a cada grupo de la siguiente manera:

En una primera sesión, los niños eran sometidos a la condición de texto presente. A cada fila se entregó un texto diferente (fila 1: TEXTD1; fila 2: TEXTN1: fila 3: TEXTD2; fila 4: TEXTN2). Los niños debían contestar a las preguntas que se formulaban en el texto, teniendo siempre el texto presente.

Una segunda sesión consistía en un intercambio de textos entre los grupos, quedando la distribución de los textos como se describe a continuación (fila 1: TEXTN1; fila 2: TEXTD1; fila 3: TEXTN2; fila 4: TEXTD2). Los niños eran sometidos a la condición de texto diferido, la cual consistía en retirar el texto una vez transcurrido el tiempo de lectura, y contestar las preguntas que se formulaban sobre el mismo.

\section{ANALISIS DE RESULTADOS}

Para determinar la influencia de la variable método de instrucción lectora, cuyos valores son: 1) global-natural, 2) silábico y 3) fónico, se tomaron como variables dependientes en los tres grupos, las puntuaciones obtenidas en las distintas habilidades de trabajo intelectual.

Estas variables se consideran según las diferentes condiciones que se plantean en el estudio.

Por consiguiente, los resultados van a ser presentados teniendo en cuenta cada una de esas condiciones. Todos los análisis se han realizado a través del programa Oneway del paquete de aplicaciones estadísticas SPSS. 


\section{1) Condición de texto presente}

En este caso, interesaba comprobar si los diferentes métodos de instrucción de la lectura había producido efectos diferenciales o no en los tes grupos para cada una de las habilidades de trabajo intelectual, en la condición de texto presente.

Los resultados de este análisis nos permite verificar que los métodos de instrucción han producido efectos significativos en TITULO $(\mathrm{F}=4.471 ; \mathrm{p}<0,01)$; RESUMEN $(\mathrm{F}=15.910 ; \mathrm{p}<0,000)$; $\mathrm{RENDIMIENTO} \mathrm{GLOBAL}(\mathrm{F}=15.038$; $\mathrm{p}<0,000)$.

Mediante la prueba de rango de Duncan, se encuentra superioridad del método global-natural, al producir diferencias a un nivel de confianza del 1 por 100 sobre las habilidades de RESUMEN y RENDIMIENTO GLOBAL, y, a un nivel de confianza del 5 por 100 , sobre la habilidad de TITULO (fig. 1).

\section{Figura 1}

Representación gráfica de los resultados obtenidos en la condición de texto presente.

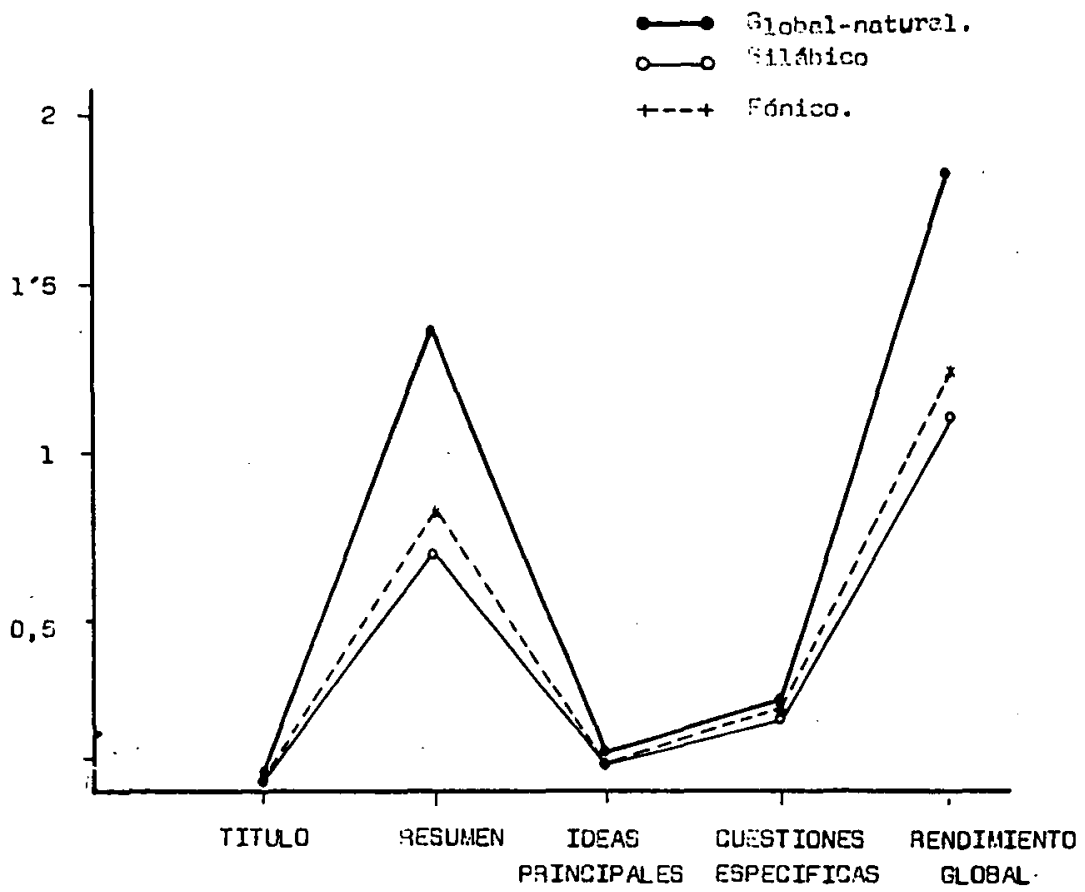

Todo ello, nos viene a indicar que los niños que han recibido un método de instrucción global-natural, a la hora de enfrentarse a la lectura de un texto, muestran un rendimiento superior cuando pueden consultar el texto.

\section{a) Texto presente descriptivo}

Teniendo en cuenta la doble condición de texto directo y naturaleza descriptiva del texto, los resultados del análisis de varianza, nos indican que los métodos de instrucción han producido efectos significativos en RESUMEN ( $F=5.860$; $\mathrm{p}<0,000)$ y RENDIMIENTO GLOBAL $(\mathrm{F}=8.214 ; \mathrm{p}<0,000)$. Al utilizar la prueba de rango de Duncan, se comprueba que el método de instrucción 
global-natural, produce efectos significativos al nivel de confianza del 5 por 100 para las habilidades de RESUMEN y RENDIMIENTO GLOBAL (fig. 2). Lo cual nos indica que los niños que han estado recibiendo este tipo de instrucción muestran un mayor rendimiento global cuando el texto es de naturaleza descriptiva, destacando también la habilidad de síntesis o resumen.

FIGURA 2

Representación gráfica de los resultados obtenidos en la condición de texto presente descriptivo

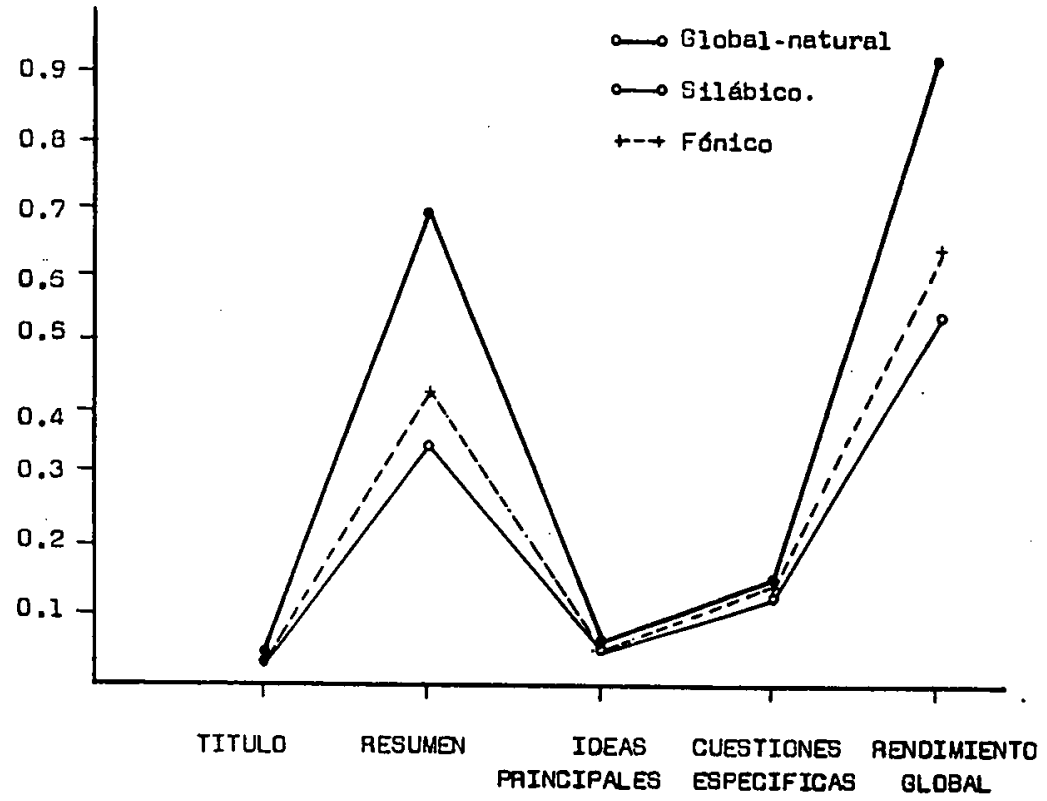

Figura 3

Representación gräfica de los resultados obtenidos en la condición de texto presente narrativo

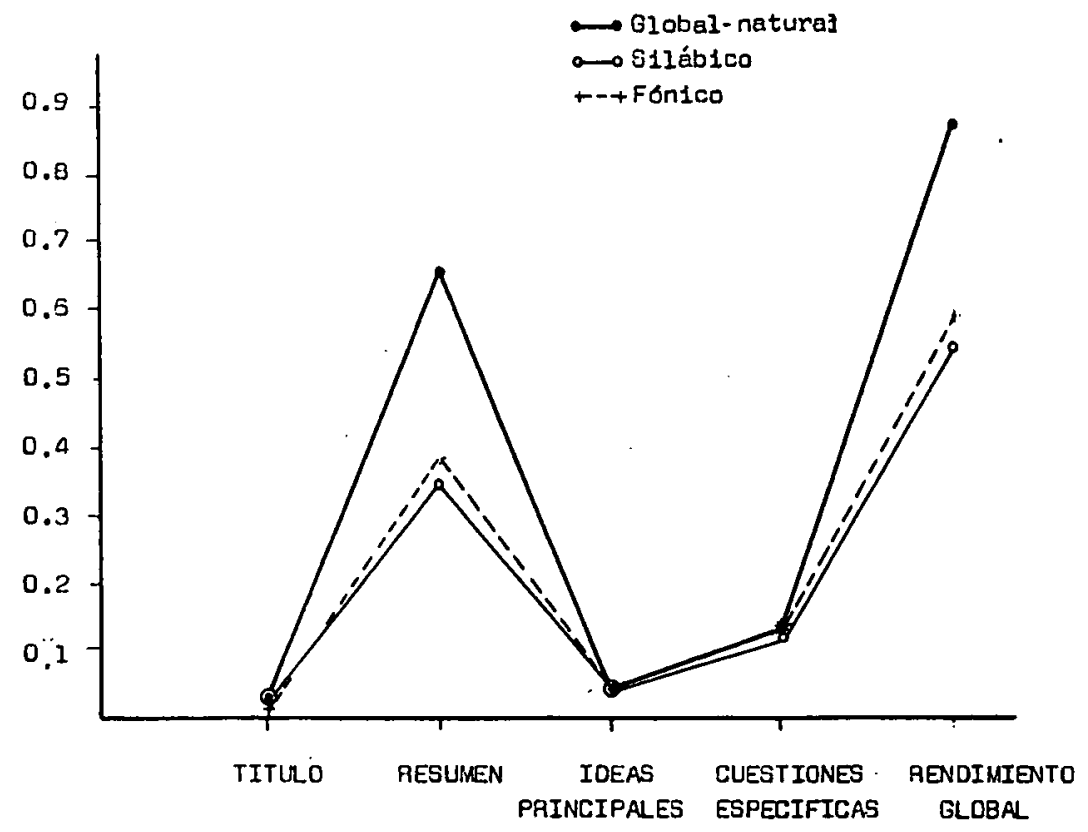


Los resultados demuestran que los métodos de instrucción han producido efectos significativos en RESUMEN ( $F=5.980$; $\mathrm{p}<0,002$ ) y RENDIMIENTO GLO$\mathrm{BAL}(\mathrm{F}=4.405 ; \mathrm{p}<0,01)$. Se desprende de esos datos, la existencia de efectos significativos producidos por los diferentes métodos de instrucción lectora sobre el rendimiento global y habilidad de resumen. Según la prueba de Rango de Duncan, el método global-natural produce efectos significativos al nivel de confianza del 1 por 100 para las habilidades de RESUMEN y, a un nivel de confianza del 5 por 100, para la variable de RENDIMIENTO GLOBAL (fig. 3).

\section{2) Condición de texto diferido}

Los resultados demuestran que los métodos de instrucción han producido efectos significativos en TITULO ( $\mathrm{F}=4.868 ; \mathrm{p}<0,008)$; RESUMEN ( $\mathrm{F}=15.240$; $\mathrm{p}<0,000)$; IDEAS PRINCIPALES $(\mathrm{F}=3.785 ; \mathrm{p}<0,02)$ y RENDIMIENTO GLOBAL $(F=13.993 ; p<0,000)$, siendo el método global-natural el que produce diferencias a un nivel de confianza del 1 por 100 sobre las habilidades de RESUMEN y RENDIMIENTO GLOBAL. Y, a un nivel de confianza del 5 por 100, sobre la habilidad de IDEAS PRINCIPALES (fig. 4).

\section{Figura 4}

Representación gráfica de los resultados obtenidos en la condición de texto diferido

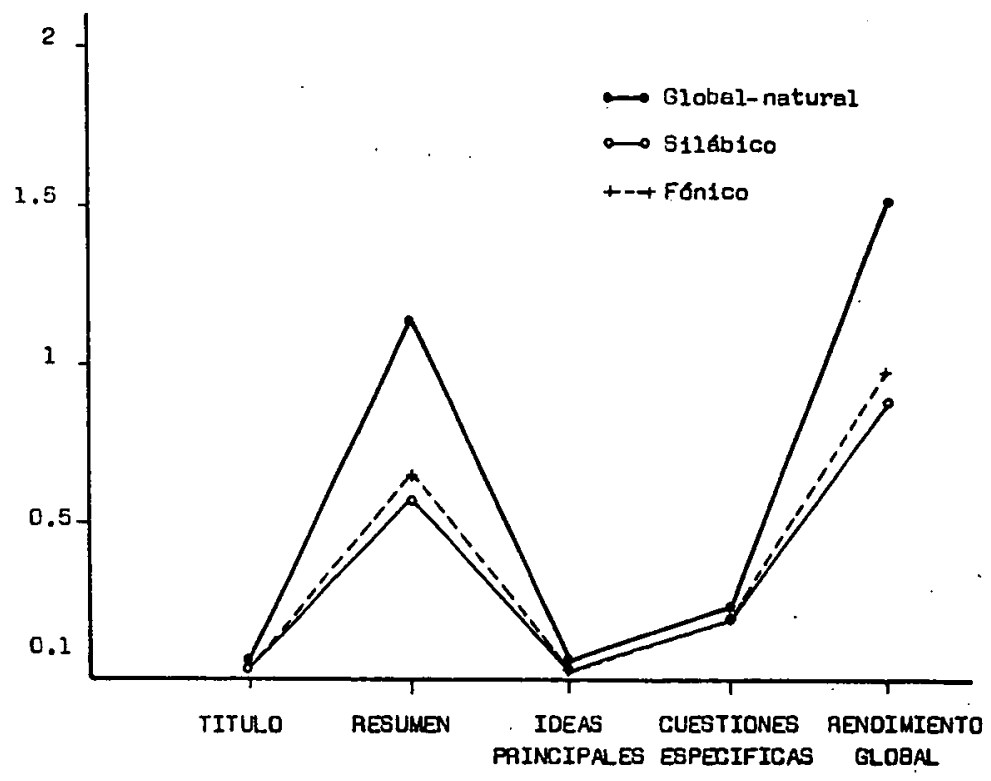

a) Texto diferido descriptivo

Los resultados del análisis de varianza demuestran, en esta ocasión, que los métodos de instrucción lectora no han producido efectos significativos.

\section{b) Texto diferido narrativo}

El análisis de varianza arroja resultados significativos respecto a las variables de RESUMEN $(\mathrm{F}=10.096 ; \mathrm{p}<0,000)$ y RENDIMIENTO GLOBAL 
$(\mathrm{F}=8.265 ; \mathrm{p}<0,000)$. Según la prueba de Rango de Duncan, el método global-natural produce diferencias a un nivel de confianza del 1 por 100 sobre las habilidades de RESUMEN y RENDIMIENTO GLOBAL (fig. 5).

\section{Figura 5}

Representación gráfica de los resultados obtenidos en la condición de texto diferido narrativo

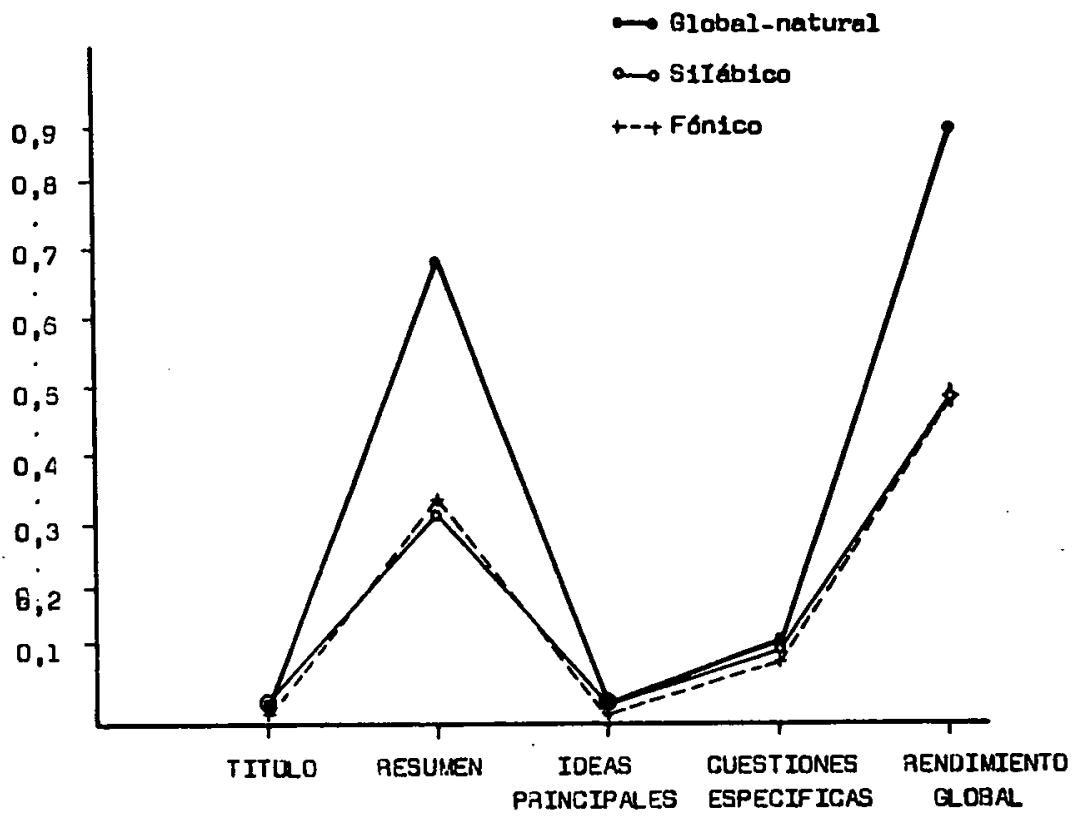

\section{DISCUSION Y CONCLUSIONES}

A la vista de los hallazgos obtenidos, es de destacar la superioridad del método global-natural frente a los métodos instruccionales restantes, al producir niveles de rendimiento global lector superiores en cada una de las condiciones consideradas en el estudio, excepto para la condición de texto diferido descriptivo, donde ninguno de los métodos instruccionales llegan a producir diferencias.

Esto significa que los escolares que han recibido el método de instrucción global-natural, muestran mejor capacidad de trabajo intelectual durante el proceso lector.

Si consideramos los resultados hallados en cada una de las condiciones en relación con las capacidades evaluadas, sobresale la habilidad de resumen o síntesis, como la habilidad sobre la cual ejerce mayor influencia el método de instrucción global-natural. Y, a ello, habría que añadir también, la habilidad de extraer las ideas principales del texto cuando éste no está presente.

En cambio, ninguno de los grupos difieren en la habilidad de responder a cuestiones específicas del texto, lo cual viene a confirmar resultados de investigaciones anteriores acerca de la no existencia de diferencias en comprensión lectora específica según los distintos métodos de lectura, cuando ha transcurrido un tiempo de instrucción (Williams, 1965; Bond y Dykstra, 1967; Chall, 1979; Gibson y Levin, 1975).

La literatura de investigación sobre este tema, ha demostrado cómo la adquisición de las estrategias de decodificación grafo-fónica o del significante, se pro- 
duce con mayor rapidez en los métodos que enfatizan, desde el principio, la decodificación del significante o procesos «Bottom-up», como son los métodos fónico y silábico.

Esto último, es lógico debido a la insistencia de estos métodos por automatizar el conocimiento de los signos, y, la habilidad para decodificarlos (LaBerge y Samuels, 1974). Sin embargo, los escolares que aprenden por un método global, al final, se equiparan al resto en esta habilidad.

Todo ello implica que los objetivos de tipo reproductivo se consiguen con cualquiera de los métodos; sin embargo, los escolares sometidos a un método de instrucción que enfatiza el significado, o, en términos cognitivos, los procesos sTop-downs en el aprendizaje de la lectura, como es el método global-natural, se caracterizan por presentar hábitos intelectuales, durante el proceso lector, de tipo productivo (Hernández, 1983).

Esto parece coincidir también con los resultados hallados por las distintas investigaciones realizadas en el campo de la psicolingüistica. La investigación psicolingüística actual, como señala Moxley (1974), apoya el principio de que la lectura es un proceso, guiado por el significado (Goodman, 1970; Langer y SmithBurke, 1982), interactivo, de múltiples niveles y generador de hipótesis (Spiro, Bruce y Blewer, 1980).

En este sentido, adquiere especial importancia las experiencias que el lector utiliza para la construcción de una representación con significado del texto. Su conocimiento previo, organizado en esquemas, ofrece un contexto para la comprensión (Anderson, 1977; Morgan, 1983; Spiro, Bruce y Blewer, 1980).

Por consiguiente, la superioridad manifiesta de los escolares que aprenden a leer por un método global-natural, constituye un indicio de que estos escolares han estado aprendiendo a leer por un método que enfatiza los procesos \&Topdown», y el significado, de ahí, que la lectura esté siendo aprendida como proceso activo, constructivo e inferencial.

Esto no quita para valorar que los métodos fónico y silábico tienden a desarrollar más las capacidades relacionadas con la memorización y la capacidad discriminativa de los escolares, según resultados de otro estudio llevado a cabo dentro de esta misma investigación (Jiménez y Hernández, 1985).

En síntesis, de todos estos resultados se derivan implicaciones importantes para la enseñanza de la lectura. Y, es concretamente, la importancia que tiene el trabajar la codificación y decodificación, relacionando los aspectos del significante (fonemas y grafemas constituidos en palabras) con la realidad representada de forma contextual. Estas características contextuales, son las que identifican al método natural y global, siendo los procesos de codificación y decodificación más ricos e integrados.

\section{Resumen}

Este estudio consistió en investigar la influencia de los métodos de lectura en la capacidad de trabajo intelectual de los escolares durante el proceso lector. Concretamente, métodos que ponen énfasis en los procesos de decodificación del significante (p. e., fónico y silábico) frente a métodos que trabajan los procesos aTop-down» o mayor énfasis en el significado ( $p$. e., global-natural).

Para ello, se analizó sobre una muestra de 565 escolares, de ambos sexos, pertenecientes a distintos estratos socioeconómicos y colegios públicos y privados, el uso que los escolares bacian de la información durante la lectura $y$ estudio de textos descriptivos y natrativos.

Se tuvo en cuenta habilidades como poner un título, realizar una sintesis o resumen, extraer las ideas principales y responder a cuestiones especificas del texto.

Los resultados demuestran que el método global-natural contribuye al desarrollo de una mejor capacidad de trabajo intelectual, manifeständose, sobre todo, en la capacidad de resumir y de extraer las ideas principales cuendo el texto no está presente. 


\section{Summary}

This paper points to give information about influence of reading methods on the intellectual work capacity of students during the reading processes. Methods that emphasize the processes of significant decodification (e. g., phonic and syllabic) versus methods wich work the sTop-down processes or emphasize more the meaning (e. g., global-natural).

The use of information while reading and studying descriptive and narrative texts, were analyzed on a sample of 565 students of both sexes, from different socio-economic status, belonging to public and private schools.

Skills so as to be able to put a title, to make a synthesis or summary, to extract the main ideas and to answer to specific cuestions from text, were taken into account.

The outcomes show the global-natural method contributes to the development of a better capacity for intellectual work, which appears, specially, in the capacity for synthesis and stracting the main ideas when the text is not present.

\section{Referencias}

AIvERMANN, D. y BOOTHBY, P. (1982). Text differences children's perceptions at the transition stage in reading. The Reading Teacher, 36; 298-302.

ANDERSON, R. (1977). Schema-directed processes in language comprehension. Technical report, N. 50, Urbana, III: University of Illinois, Center for the study of reading.

BOND, G. y DYKSTRA, R. (1967). The cooperative research program in first-grade reading instruction. Reading Research Quarterly, 2; 5-142.

CHAL, J. (1967). Learning to read: The great debate. Nueva York: McGraw-Hill.

ChAll, J. (1979). The great debate: Ten years later, with a modest proposal for reading stages. En L. B. Resnick \& P. A. Weaver (Eds.) Theory and practice of early reading, Vol. 1, Hillsdale, N. J.: Erlbaum.

GiBson, E y LEVIN, H. (1975). The psychology of reading. Cambridge, Mass: Mit Press.

Goodman, K. (1970). Reading: A psycholinguistic guessing game. En Harry Singer y Robert G. Ruddell (Eds.), Theoretical models and processes of reading. Newark, Del: International Reading Association.

HERNÁNDEZ, H. (1983). Procesos de decodificación de aAbajo-arriba» y de aArriba-abajos en la lectura. Revista de Investigación Psicológica, Vol. 1, núm. 0; 137-164.

HERNÁNDEZ, P. y GARCIA. L. (1983a). Objetivos educacionales y perfiles cognitivos en la evaluación académica. Memoria de licenciatura. Universidad de La Laguna.

JIMENEZ, G. y HERNÁNDEZ, P. (1985). Métodos de lectura y häbitos intelectuales. Tesis Doctoral. Universidad de La Laguna.

JimÉNEZ, J. y HERNÁNDEZ. P. (1986). Reading methods and intellectual abilities. Comunicación presentada al 21st International Congress of Applied Psychology, Israel.

KENT, C. (1984). A linguistic compares narrative and expository prose. Joumal of Reading, Vol. 28, 3; 232-236.

LABERGE, D. y SAMUELS, S. (1974). Toward a theory of automatic information processing in reading. Cognitive Psycho$\log y, 6 ; 293-323$.

LANGER, J. y SMITH. B. (1982). Reader meets author/bridgin the gap. Newark, Del: International Reading Association. MORGAN, A. (1983). Context: The web of meaning. Languaje Arts, Vol. 60; 305-313.

MOXuEY, L. (1984). The compositional approach to reading in practice and theory. The Journal of Reading, Vol. 27,$7 ; 636-643$.

OTTO, W.; WARRET, T; SMITH, R.; DULIN, K. y JOHNSON, D. (1971). Summary and review of investigations relating to reading, July 1, 1969 to June 30, 1970. Joumal of Educational Research, Vol. 64, 6; 242-268.

OTTO, W.; BARRETT. T.; SMITH, R.; DULIN, K. y JohNSON, D. (1972). Summaty and review of investigations relating to reading, July 1, 1970 to June 30, 1971. Journal of Educational Research, Vol. 65, 6; 242-272.

OTTO. W.; BARRETT, T.; SMITH, R.; DUUN, K. y JohNSON. D. (1973). Summary and review of investigations relating to reading, July 1, 1971 to June 30, 1972. Joumal of Educational Research, Vol. 66, 8; 338-362.

PflaUm. S.; W ALBERG, H.; KAREgIaNES, M. y RACHER, S. (1980). Reading instruction: A quantitative method. Educational Researcher, Vol. 9, 7; 12-18.

ROSENSHINE, B. (1980). Skills hierarchies in reading comprehension. En Spiro, R.; Bruce, B. y Brewer, R. Theoretical issues in reading comprebension. Lawrence-Erlbaum Associates Publishers.

SPIRO, R.; BRUCE, B. y BREWER, B. (1980). Theoretical issuer in reading comprehension. Lawrence-Erlbaum Associates Publishers.

WeinTraub. S.; Robinson, H.; SMITH. H. y Plessas. G. (1971). Summary of investigations relating to reading. July 1, 1969 to June 30, 1970. Reading Research Quarterly, Vol. 6, 2; 135-319.

WEINTRAUB, S.; ROBINSON, H.; SMITH, H. y ROSER, N. (1973-74). Summary of investigations relating to reading, July 1, 1972 to June 30, 1973. Reading Research Quarterly, Vol. 9, 3; 247-513.

WWIINTAUB, S.; ROBINSON, H.; SMITH, H.; PIESSAS. G. y Rowls, M. (1974-75). Summary of investigations relating to reading: July 1, 1973, to June 30, 1974. Reading Researchy Quarterly, Vol. $103 ; 543 \mathrm{p}$.

WEINTRAUB, S.; SMITH, H.; ROSER, N.; KIBBY, M.; MOORE, W. y BECK, F. (1982). Summary of investigations relating to reading July 1, 1980 to June 30, 1981. Newark, Del.: International Reading Association.

WIUIMMS. J. (1965). Reading research and instruction. Review of Educational Research, Vol. 35; 147-153. 


\section{TEXTN 1}

Texto narrativo primero

La excursión de Marcose

Marcos fue al Teide con sus padres y bermanos para ver la nieve. En medio de la nieve habia unas rocas que brillaban. Marcos fue corriendo hacia alli. Se estretuvo contemplando los colores de las rocas.

Luego, quiso volver donde estaba su familia, pero se dio cuenta de que estaba perdido. Se puso a llorar, pero de pronto se dio ánimos a sí mismo y se subió a lo alto de una montaña. Se quitó la camisa y comenzó a sacudirla en el uire dando gritos.

Entonces, aparecieron unos montañeros que lo llevaron basta sus padres.

TEXTN2

Texto narrativo segundo

¿El pez BOTI»

En una hermosa mañana de verano nació un pez que llamaron Boti. Sus aletas eran de color verde y tenía unos grandes ojos azules.

Boti era muy despistado, por eso nunca se separaba de sus bermanos. Todos los dias paseaban por el fondo del mar. Unas veces iban de excursión a un sitio donde habia unas rocas muy grandes, y otras veces a visitar una playa.

Boti tenía un deseo. Era poder volar sobre el mar. Un día encontró una pequeña cajita de cristal en la orilla de la playa. Cuando la abrió se encontró unas alas grandes. Al verlas, Boti se las puso y se echó a volar. Así se cumplió su deseo.

TEXTD 1

Texto descriptivo primero "La máquina TOBO"

Tobo es una máquina que sirve para la limpieza de las ciudades. En la parte delantera tiene unos faros luminosos. Los faros son de color rojo y se encienden solamente por la noche. De día permanecen apagados.

Debajo de la máquina hay unas ruedas peludas. Estas ruedas son como escobas que bamen la basura de las calles. Encima de la máquina bay unos tubos largos. Por esos tubos sale agua a presión que deja la calle mojada.

En el interior de la máquina hay un depósito. En él se queda toda la basura que es recogida.

TexrD2

Texto descriptivo segundo

"El palacio de las bormigas*

Las hormigas ban construido un palacio de azúcar debajo de la tierra. El palacio tiene unos grandes balcones. Los balcones son de mantequilla, tienen un color amarillo y están adormados con puntitos de mermelada. En lo alto del palacio hay una torre de caramelo.

Al lado del palacio, las hormigas tienen una gran piscina formada por una cáscara llena de agua. El agua de la piscina es transparente y tiene un color azul.

Existe también una fäbrica que las hormigas utilizan para triturar los alimentos. Estos alimentos son colocados debajo de unas tapas de botella para protejerlos del frío. 
TEXTN1

Hoja de preguntas correspondiente al texto narrativo primero

- Ponle un título al cuento:

- Escribe un resumen del cuento que has leido:

- Escribe las tres cosas más importantes que dice el cuento:

- ¿Con quién fue Marcos al Teide?:

- ¿Cómo eran las rocas que vio Marcos?:

- Cuando Marcos se perdió, ¿qué bizo antes de subir a la montaña?

- ¿Qué hizo Marcos con su camisa?:

- ¿Qué ocurrió al final del cuento?:

\section{TEXIN2}

Hoja de preguntas correspondiente al texto narrativo segundo

- Ponle un título al cuento:

- Escribe un resumen del cuento que bas leido:

- Escribe las tres cosas más importantes que dice el cuento:

- ¿Cuándo nació Boti?:

- ¿Cómo es el cuerpo de Boti?:

- ¿Qué hacía Boti todos los días?

- ¿Qué había en la cajita de cristal?

- Cuando Boti encontró la cajita de cristal, ¿qué bizo?:

TEXTD1

Hoja de preguntas correspondiente al texto descriptivo primero

- Ponle un título al cuento:

- Escribe un resumen del cuento que bas leído:

- Escribe las tres cosas más importantes que dice el cuento:

- ¿Para qué sirve la máquina Tobo?:

- ¿Cómo son los faros de la máquina?:

- ¿Qué hacen las ruedas peludas de la máquina?

- ¿Por dónde pasa el agua que la máquina expulsa a la calle?:

- ¿Para qué sirve el depósito de la máquina?:

TexTD2

Hoja de preguntas correspondiente al texto descriptivo segundo

- Ponle un título al cuento:

- Escribe un resumen del cuento que has leído:

- Escribe las tres cosas más importantes que dice el cuento:

- ¿Dónde construyeron las hormigas el palacio?:

- ¿Cómo son los balcones del palacio?:

- ¿Qué hay en lo alto del palacio?

- ¿Cómo es la piscina de las hormigas?:

- ¿Qué hacen las bormigas con los alimentos que trituran?: 